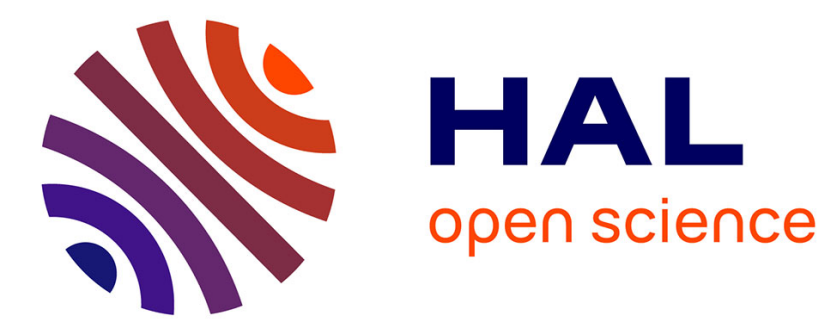

\title{
Magnetoelastic modelling in soft nanocrystalline alloys
}

Hervé Chazal, J.L. Porteseil, Olivier Geoffroy, T. Waeckerle, Francisco Alves

\section{To cite this version:}

Hervé Chazal, J.L. Porteseil, Olivier Geoffroy, T. Waeckerle, Francisco Alves. Magnetoelastic modelling in soft nanocrystalline alloys. Journal of Magnetism and Magnetic Materials, 2006, 304 (2), pp.145-149. 10.1016/j.jmmm.2006.02.123 . hal-00192192

\section{HAL Id: hal-00192192 \\ https://hal.science/hal-00192192}

Submitted on 14 Jan 2020

HAL is a multi-disciplinary open access archive for the deposit and dissemination of scientific research documents, whether they are published or not. The documents may come from teaching and research institutions in France or abroad, or from public or private research centers.
L'archive ouverte pluridisciplinaire HAL, est destinée au dépôt et à la diffusion de documents scientifiques de niveau recherche, publiés ou non, émanant des établissements d'enseignement et de recherche français ou étrangers, des laboratoires publics ou privés.

\section{(c)(1)}

Distributed under a Creative Commons Attribution| 4.0 International License 


\title{
Magnetoelastic modelling in soft nanocrystalline alloys
}

\author{
Geoffroy Olivier ${ }^{\mathrm{a}, *}$, Chazal Hervé ${ }^{\mathrm{a}, \mathrm{b}, \mathrm{c}}$, Porteseil Jean-Louis ${ }^{\mathrm{a}}$, \\ Waeckerlé Thierry ${ }^{\mathrm{c}}$, Alves Francisco ${ }^{\mathrm{d}}$ \\ ${ }^{\mathrm{a}}$ Laboratoire Louis Néel, CNRS, BP 166, 38042 Grenoble, France \\ ${ }^{\mathrm{b}}$ Laboratoire d'Electrotechnique de Grenoble, BP 46, F-38402 saint Martin d'Hères cedex, France \\ ${ }^{\mathrm{c}}$ Imphy Alloys, 58160 Imphy, France \\ ${ }^{\mathrm{d}}$ LGEP, SUPELEC, Plateau du Moulon, 91192 Gif sur Yvette, France
}

\begin{abstract}
Magnetoelastic effects in ultra soft nanocrystalline alloys are investigated theoretically and experimentally. From $H_{\mathrm{c}}$ measurements, extraction of magnetoelastic contribution is carried out using a formalism obtained revisiting random anisotropy model (RAM) in the light of domain walls (DW) displacements, our approach based on theoretical investigations on the way of a reversal of a correlated volume $(\mathrm{CV})$ located in the vicinity of a DW. Modelling of magnetoelastic effects shows that even in perfectly relaxed samples, a magnetoelastic contribution exists due to elastic frustration experienced by a CV during its magnetization reversal. Magnitude of this energy is large enough to drive coercivity of samples featuring grain diameter $D$ around $10 \mathrm{~nm}$, which are of major interest for applications.
\end{abstract}

Keywords: Nanocrystalline alloys; Magnetoelastic effects; Random anisotropy

\section{Introduction}

Magnetoelastic effects in soft nanocrystalline alloys are of major importance. Experimental data show that it is necessary to consider, apart from classical random anisotropy contribution, a second source of coercitivity usually attributed to magnetoelastic effects [1,2]. Until now, the involved mechanisms are unclear and the present work proposes to answer this question.

Magnetoelastic coercivity results due to internal frustration and can be investigated at different scales. At first, one can look to the frustration occurring at the frontier of the nanocrystal and surrounding amorphous, these media have different magnetostriction coefficients $\lambda_{\mathrm{a}} \approx 22 \times 10^{-6}$ (amorphous), $\lambda_{\mathrm{c}} \approx-5 \times 10^{-6}$ (FeSi nanocrystals) [3-5]. At the scale of the so-called correlated volume (CV), characterized by dimensions comparable to the ferromagnetic correlation length $L_{0}$, this effect is subjected to the same averaging process as the magnetocrystalline aniso-

\footnotetext{
*Corresponding author. Tel.: + 33476887904 ; fax: + 33476881191 . E-mail address: geoffroy@grenoble.cnrs.fr (G. Olivier).
}

tropy and leads to a negligible contribution compared with the magnetocrystalline one [6]. This allows to consider the ribbon as isotropic and elastically homogeneous, with magnetostriction coefficient $\lambda_{\mathrm{s}}$ and Young Modulus $E$ leading to a magnetoelastic energy density $W_{\mathrm{me}} \approx E \lambda_{\mathrm{s}}^{2}$. With $\lambda_{\mathrm{s}} \approx$ several $10^{-6}$ and $E \approx 2 \times 10^{11} \mathrm{~Pa}$, one obtains $W_{\mathrm{me}} \approx$ several $\mathrm{J} / \mathrm{m}^{3}$, which, dealing with macroscopic properties, can play a role only if coherent at the scale of the CV.

Looking for a mechanism able to induce such a coherent stress, we consider the reversal of the magnetization in a CV. A mismatch arises from its immersion in a medium differently magnetized, maximum frustration occurring when the magnetizations are perpendicular to each other. The magnetoelastic effects will be those of a coherent uniaxial anisotropy, denoted in the following as $K_{\text {me }}$. A quantitative modelling of $K_{\mathrm{me}}$ is therefore of great interest and proposed in Section 3.

Experimental data concern ribbons (atomic composition $\mathrm{Fe}_{75.3} \mathrm{Cu}_{1} \mathrm{Nb}_{2.84} \mathrm{Si}_{14.6} \mathrm{~B}_{6.2}$, thickness $\approx 21 \mu \mathrm{m}$ ) manufactured by Imphy Alloys. The ribbons are annealed at different temperatures $490<T<570^{\circ} \mathrm{C}$ and durations $1<t<600 \mathrm{~min}$. 
Crystalline fraction $f$ and grain diameter $D$ are characterized by X-ray diffraction. The large database (30 samples) obtained feature $8<D<13 \mathrm{~nm}$ and $10<f<75 \%$. Confrontation with the modelling is based on $H_{\mathrm{c}}$ measurements. The part due to random anisotropy being not negligible, the extraction of magnetoelastic contribution to $H_{\mathrm{c}}$ involves dedicated formalism as proposed in Ref. [3]. We propose, in part 2, an alternative formalism considering $\mathrm{CV}$ reversal in a macroscopic scheme governed by domain wall (DW) displacement. Extraction of $K_{\mathrm{me}}$ from experiments will be then carried out, allowing confrontation with modelling, marking the end of part 3.

\section{CV reversal and DW displacement: Application to the extraction of experimental $K_{\mathrm{me}}$}

In the frame of random anisotropy model (RAM), the basic acting entity is the CV. Since the exchange stiffness $A$ prevents changes in the direction of magnetization within very short distance, the magnetization mechanism is assumed to be of the uniform rotation. As a result, the anisotropy of an assembly of nanograins, settling $\mathrm{CV}$, is the average of individual contributions, leading, for randomly oriented ferromagnetically coupled grains, to an effective anisotropy $K_{\mathrm{e}}$ much lower than the intrinsic magnetocrystalline anisotropy $K_{0}$ featured by one individual nanograin. The effective anisotropy governs the reversal field $H_{\mathrm{r}} \approx$ $2 K_{\mathrm{e}} / J_{\mathrm{s}}\left(J_{\mathrm{s}}=\right.$ saturation polarization $)$, associated to the $\mathrm{CV}$ and thus the resulting coercivity [3-5].

The preceding scheme considers the $\mathrm{CV}$ as an isolated particle. In fact, from a macroscopic point of view, the magnetization variation is due to DW displacements. In other words, an acting CV behaves in a co-operative way with the neighbours by the way of magnetostatic interactions. Our goal is thus to determine the quantitative influence of these interactions upon $H_{\mathrm{r}}$ and, at a larger scale, upon $H_{\mathrm{c}}$.

To do this, we will consider a peculiar $\mathrm{CV}$, denoted $\mathrm{CV}_{0}$, and neighbouring $\mathrm{CVs}$, those acting as a global entity, that is the DW. In this frame, $\mathrm{CV}_{0}$ is considered as a pinning centre with $H_{\mathrm{r} 0}$ as pinning field. Looking to the evolution of the interaction energy $E\left(z_{\mathrm{d}}\right)$ with $z_{\mathrm{d}}, z_{\mathrm{d}}$ denoting the relative position $\mathrm{CV}_{0} / \mathrm{DW}$, allows to determine $H_{\mathrm{r} 0}$. Finally and according to Ref. [7], $\left\langle\left\langle\mathrm{H}_{\mathrm{r}}\right\rangle\right\rangle$ obtained by averaging $H_{\mathrm{r} 0}$ on the population of $\mathrm{CV}$ s coinciding with the DW, will be directly related to $H_{\mathrm{c}}$ as

$H_{\mathrm{c}}=\overline{\ll H_{\mathrm{r}} \gg}+{\overline{\left(\ll H_{\mathrm{r}} \gg-\overline{\ll H_{\mathrm{r}} \gg}\right)^{2}}}^{1 / 2}$

(Horizontal bars indicate an average process on the different absolute positions of the wall). Usually, $E\left(z_{\mathrm{d}}\right)$ exhibits even parity, leading, with defects uniformly distributed, to $\overline{\left\langle H_{\mathrm{r}} \gg\right.}=0$ [7]. $H_{\mathrm{c}}$ is so determined by the second term of Eq. (1). As we shall see, the situation corresponding to our case is different.

The first step is to determine $E\left(z_{\mathrm{d}}\right)$. To do this, we picture the DW by a region of thickness $L_{0}$ (Fig. 1). Magnetiza-

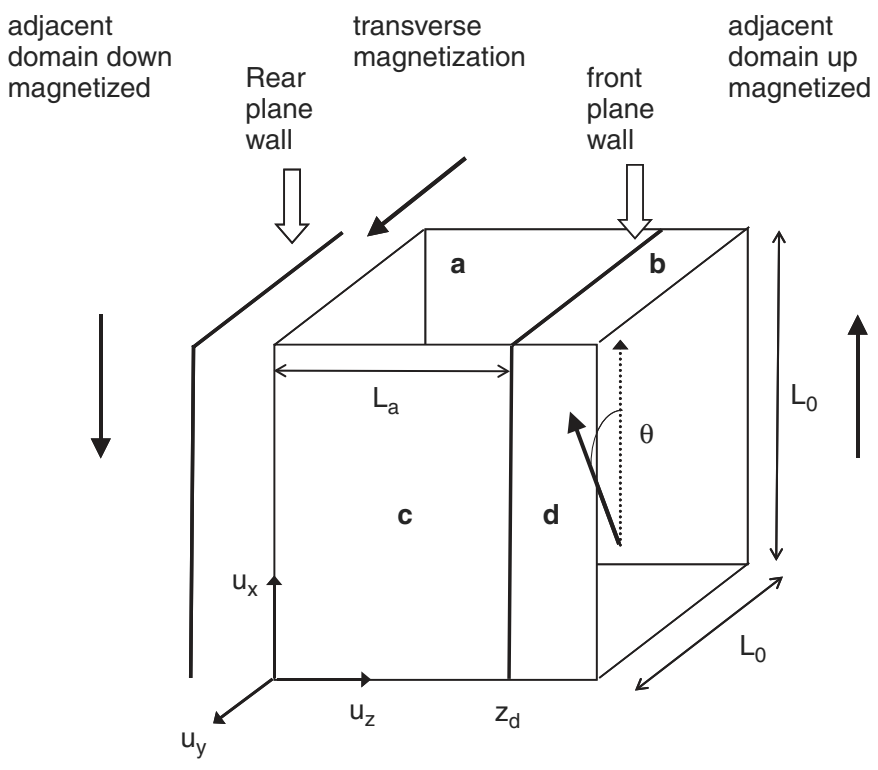

Fig. 1. Interaction of $\mathrm{CV}_{0}$ with the surrounding $180^{\circ} \mathrm{DW}$. Magnetization of $\mathrm{CV}_{0}$ is assumed uniform, its direction included in the DW plane characterized by $\theta\left(z_{\mathrm{d}}\right)$

tions of $\mathrm{CV}$ and surrounding media (DW or adjacent domains (AD)) are different, leading to a magnetostatic energy $E_{\mathrm{m}}$. For convenience, magnetization of different media are assumed uniform, the DW being transversally magnetized. We obtain the uniform densities of the magnetic charges deposited on faces $a, c$ (part of $\mathrm{CV}_{0}$ surrounded by DW), and $b, d$ (part of $\mathrm{CV}_{0}$ surrounded by $\mathrm{AD}$ ), and opposite faces. $E_{\mathrm{m}}$ thus involves self-contributions $E_{\mathrm{m} i}$ corresponding to rectangles $a_{i}$-sections $L_{\mathrm{a}} \times L_{0}$ or $\left(L_{0}-L_{\mathrm{a}}\right) \times L_{0}$ - with charge density $\sigma_{i}$, and interaction terms $E_{\mathrm{m} i j}$ (calculated with centred charges instead of charge densities). $E_{\mathrm{m} i}$ is given as follows [9]:

$$
\begin{aligned}
4 \pi \mu_{0} E_{\mathrm{m} i}= & L_{0}^{3} \sigma_{i}^{2} F\left[p_{i}\right], \quad p_{i}=L_{a} / L_{0} \text { or }\left(L_{0}-L_{\mathrm{a}}\right) / L_{\mathrm{a}} \\
F[p]= & p^{2} \operatorname{Ln}\left[1 / p+\sqrt{1+1 / p^{2}}\right]+p \operatorname{Ln}\left[p+\sqrt{1+p^{2}}\right] \\
& +\left[1+p^{3}-\left(1+p^{2}\right)^{3 / 2}\right] / 3 .
\end{aligned}
$$

In the domain of interest $\left(0<p_{i}<1\right), F$ accepts - and will be replaced by - a more easily analytically tractable approximation function $\mathrm{G}$ defined as

$$
G[p]=a p^{2}-b p^{3} ; \quad a=2.58525, \quad b=1.13266 .
$$

With $E_{k}=K \sin ^{2} \theta$, denoting the CV volumetric density of anisotropic energy, we write the total reduce energy $E_{\mathrm{r}}=$ $\left(E_{\mathrm{m}}+E_{k}\right)_{\mathrm{r}}$ using dimensionless parameters $k=K \pi \mu_{0} / J_{\mathrm{s}}^{2}$ and $u=z_{\mathrm{d}} / L_{0}$, as

$$
\begin{aligned}
E_{r}= & k \sin ^{2} \theta+(1-\cos \theta-\sin \theta) u(1-u)(1-1 / \sqrt{5}) \\
& +(1-\cos \theta)\left[(a-1 / 2)(1-u)^{2}-b(1-u)^{3}\right] \\
& +(1-\sin \theta)\left[(a-1 / 2) u^{2}-b u^{3}\right], \quad 0<u<1 .
\end{aligned}
$$


Symmetric formula is obtained for $1<u<2$. Minimizing $E_{\mathrm{r}}$ with respect to $\theta$, one obtains equilibrium angle $\theta_{\mathrm{e}}\left(z_{\mathrm{d}}\right)$ and equilibrium energy $E_{\mathrm{e}}\left(z_{\mathrm{d}}\right)=E_{\mathrm{r}}\left(\theta_{\mathrm{e}}\left(z_{\mathrm{d}}\right)\right)$, plotted on Fig. 2 .

The $k=0$ curve shows that, dealing with magnetostatics $\theta_{\mathrm{e}}(0)=0, \theta_{\mathrm{e}}\left(L_{0}\right)=\pi / 2$ and $\theta_{\mathrm{e}}\left(2 L_{0}\right)=\pi, \theta_{\mathrm{e}}$ is characterized by a continuous evolution. CVs featuring easy magnetization along Oy show the same behaviour with $\theta_{\mathrm{e}}\left(L_{0}\right)=\pi / 2$ again. Situation of CVs featuring $k>0$ differs : with $K$ increasing, $\theta_{\mathrm{e}}\left(z_{\mathrm{d}}\right)$ lags more and more towards 0 , and $E_{\mathrm{e}}\left(z_{\mathrm{d}}\right)$ drawing nearly asymptotic curve $E_{\mathrm{r}}(0)$. If $k$ is large enough, $\theta_{\mathrm{e}}\left(L_{0}^{-}\right)$reaches a value $\theta_{0 k}<\pi / 2$. On increasing $z_{\mathrm{d}}$ slightly, the magnetization would like to reach direction $\theta_{\mathrm{e}}\left(L_{0}^{+}\right)=$ $\pi-\theta_{0 k}$, but cannot, because of the barrier featured by the costly direction $\pi / 2$. The switching thus occurs latter (cf. point $\mathrm{B}$ on curve $k=0.8$ ), breaking the symmetry of $E_{\mathrm{e}}\left(z_{\mathrm{d}}\right)$. The transition between the two behaviours occurs for a $k_{\mathrm{L}}$ value $\approx 0.5$. The point is that $\mathrm{CVs}$ featuring $k>k_{\mathrm{L}}$ give a non zero $\ll H_{r} \gg$ term in Eq. (1), and from this point of view, settle a relevant population looking to $H_{\mathrm{c}}$.

Surprisingly, $K_{\mathrm{L}}=k_{\mathrm{L}} J_{\mathrm{s}}^{2} / \pi \mu_{0}$-and related quantities-are determined by magnetostatics instead of anisotropy and are of unrealistically high magnitude. This paradox vanishes, noticing that, dealing with the magnetostatic energy, the crude assumption of uniformly magnetized regions leads to a high charges concentration and an overestimated evaluation calling upon $\mu^{*}$ effect [10], which leads to a reduction factor between 1 and $1+J_{\mathrm{s}}^{2} /\left(2 \mu_{0} K_{\mathrm{e}}\right)$ depending on the magnetizations of $\mathrm{CV}$ and surrounding medium. $K_{\mathrm{L}}$ is now controlled by the anisotropy and is much lower. Nevertheless, the reduction is obtained through the dilution of charges, which, to be effective, involves a reversing cell probably greater than the basic $\mathrm{CV}$. This shows that looking to the basic properties (volume and shape) of CVs, we cannot be unaware of the magnetostatics, justifying future investigations.

At this step, we retain only a general teaching overview of our scheme, i.e., looking to $H_{\mathrm{c}}$, the relevant population

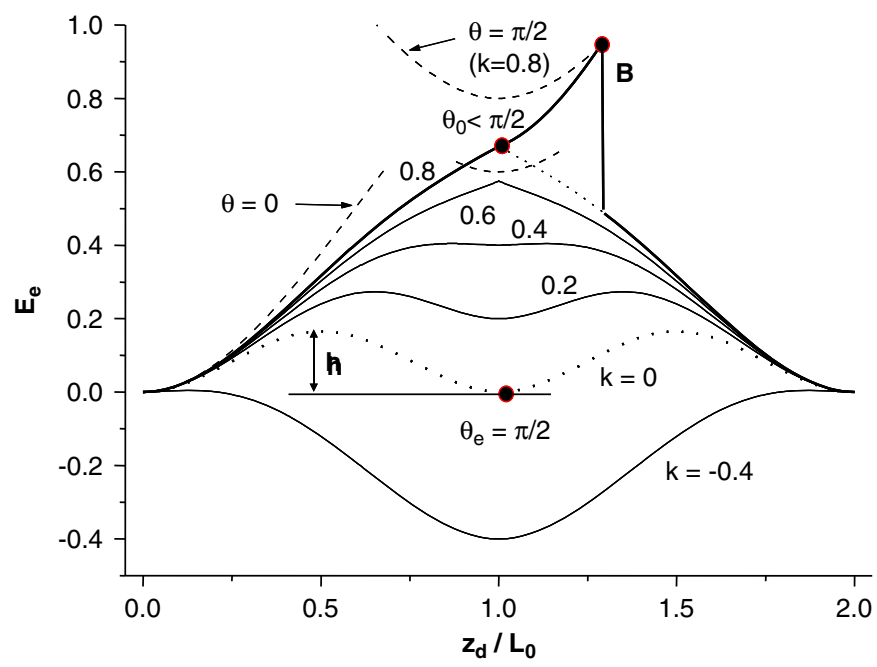

Fig. 2. Evolution of equilibrium energy of $\mathrm{CV}$ with respect to DW position for the situation described on Fig. 1. of CVs features $\left.K>K_{\mathrm{L}}\right\rangle 0$, leading to $K_{\mathrm{e}}=\langle K\rangle$. Numerical value of $K_{\mathrm{L}}$ being strongly dependent on details of reversal scheme, we take it null in the following. With a probability distribution $\mathrm{p}(K)$ Gaussian, we thus obtain.

$K_{\mathrm{e}}=\int_{0}^{\infty} K e^{-K^{2} /\left(2 \sigma^{2}\right)} d K / \int_{0}^{\infty} e^{-K^{2} /\left(2 \sigma^{2}\right)} d K=\sqrt{\frac{2}{\pi} \sigma}$

This result is comparable to the one obtained in the classical frame of RAM, our average value and usual fluctuation term both are controlled by $\sigma$.

Extending our analysis to the case of a superimposed longitudinal anisotropy $K_{\mathrm{u}}>0$ (which will correspond in our case to $K_{\mathrm{me}}$ ), $p(K)$ is now centred on $K_{\mathrm{u}}$. Increasing $K_{\mathrm{u}}$, population of CVs featuring $K<0$ progressively vanishes, leading to the asymptotic case $K_{\mathrm{e}}=K_{\mathrm{u}}$. Using dimensionless parameters $k_{\mathrm{a}}\left[K_{\mathrm{u}} /\left(f^{2} K_{0}^{4} A^{-3} D^{6}\right)\right]^{1 / 3}$ and $k_{\mathrm{e}}=K_{\mathrm{e}} /\left(K_{\mathrm{u}} k_{\mathrm{a}}\right)$, it is finally obtained [9],

$k_{\mathrm{a}}=\left[1+\sqrt{; 2 / \pi} k_{\mathrm{e}}^{3 / 4} \exp \left[-1.06 k_{\mathrm{e}}^{-0.92}\right]\right] / k_{\mathrm{e}}$,

where $k_{\mathrm{a}}$ measures respective weight of random anisotropy and $K_{\mathrm{u}}$ in the resulting $K_{\mathrm{e}} . K_{\mathrm{e}}$ calculated using Eq. (6) with typical numerical values for nanocrystalline alloys is plotted in Fig. 3. In our case, $K_{\mathrm{me}}$ plays the role of $K_{\mathrm{u}}$ and is extracted as follows.

At first, one notices that $f, K_{0}, A, D$ are known, couples $\left(K_{\mathrm{e}}, K_{\mathrm{me}}\right)$ are $k_{\mathrm{e}}$ dependent; $k_{\mathrm{e}}$ is given, $k_{\mathrm{a}}$ is deduced from Eq. (6), allowing to calculate $K_{\mathrm{e}} / K_{\mathrm{me}}$ (definition of $k_{\mathrm{e}}$ ) and $K_{\mathrm{me}}$ (definition of $k_{\mathrm{a}}$ ). From $K_{\mathrm{me}}$ and $K_{\mathrm{e}} / K_{\mathrm{me}}$, one obtains $K_{\mathrm{e}}\left(k_{\mathrm{e}}\right)$.

$K_{\mathrm{e}}$ is determined from $H_{\mathrm{c}}$ measurements provided by our database, assuming with [4] the law

$H_{\mathrm{c}}=2 \alpha K_{\mathrm{e}} / J_{\mathrm{s}} ; \quad \alpha=0.065$.

$K_{\mathrm{e}}$ known, denoted $K_{\mathrm{e} 0}$, an iterative calculation of $K_{\mathrm{e}}\left(k_{\mathrm{e}}\right)$ can be carried out, varying $k_{\mathrm{e}}$. A peculiar value $k_{\mathrm{e} 0}$ is obtained, satisfying $K_{\mathrm{e}}\left(k_{\mathrm{e} 0}\right)=K_{\mathrm{e} 0}$. The value of $K_{\mathrm{me}}$ corresponding to $K_{\mathrm{e} 0}$ is so $K_{\mathrm{me}}\left(k_{\mathrm{e} 0}\right)$.

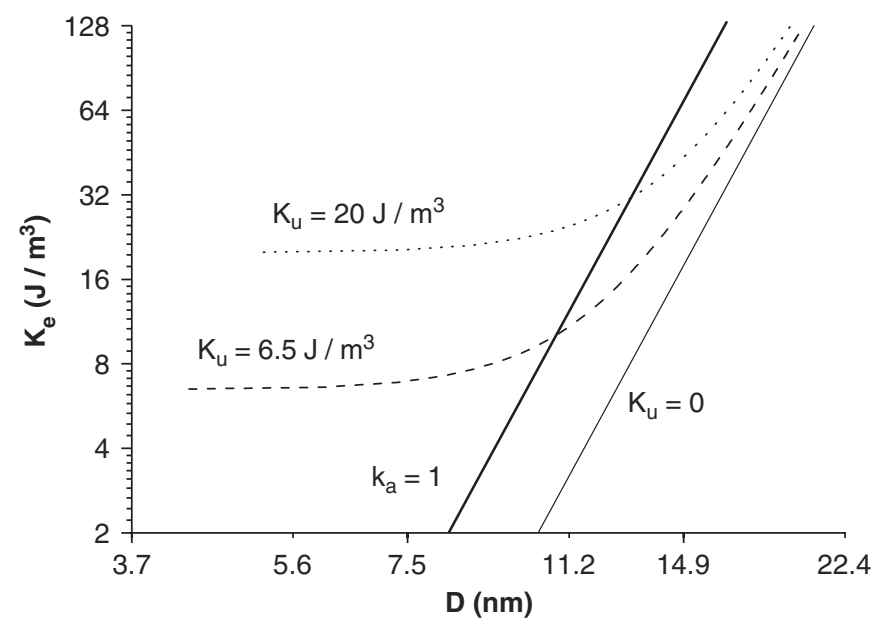

Fig. 3. Effective anisotropy $K_{\mathrm{e}}$ calculated with Eq. (6) $\left(A=10^{-11} \mathrm{~J} / \mathrm{m}\right.$, $K_{0}=8 \mathrm{~kJ} / \mathrm{m}^{3}$ ). Curve $k_{\mathrm{a}}=1$ separates regions where $K_{\mathrm{e}}$ is governed by $K_{\mathrm{u}}$ (left side) or $D$ (right side). 


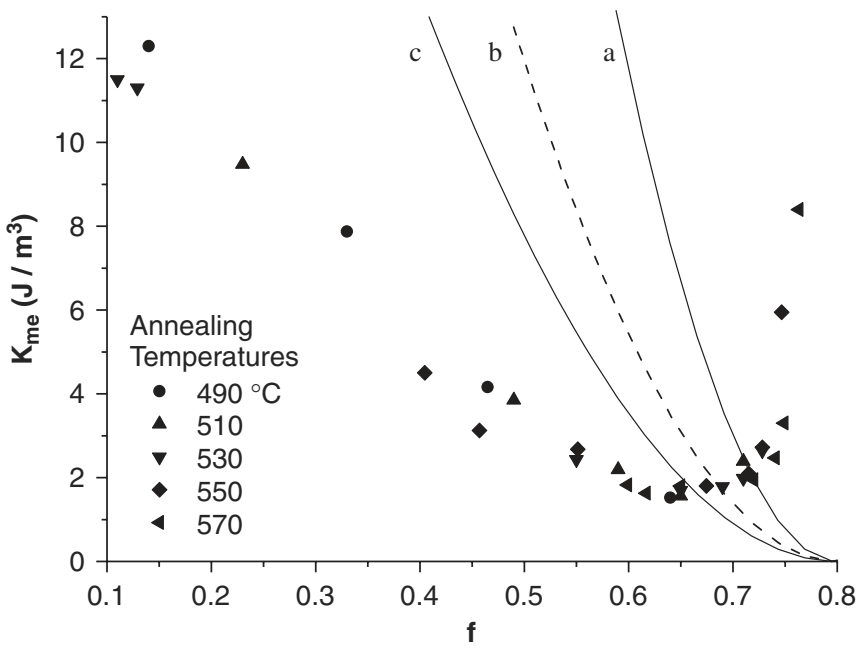

Fig. 4. Experimental values of $K_{\text {me }}$ deduced from $H_{\mathrm{c}}$ measurements using Eq. (6) and (7) and confrontation with model: (a) magnetizations along edges, no buffer zone; (b) magnetizations along edges with BZ; and (c) magnetizations along diagonals with $\mathrm{BZ}$.

Values of $K_{\mathrm{me}}$ obtained this way from $H_{\mathrm{c}}$ measurements are plotted on Fig. 4. One notices a sudden increase for $f>0.65$, corresponding to an increase of $H_{\mathrm{c}}$ as already observed in Refs. [11,12]. Of course, new phenomena occur here, which have nothing to do with magnetoelastics. Noticing that corresponding samples feature annealing treatments $\left(530^{\circ} \mathrm{C}, t>60 \mathrm{~min}\right),\left(550^{\circ} \mathrm{C}, t>10 \mathrm{~min}\right)$ or $\left(570{ }^{\circ} \mathrm{C}, t>2 \mathrm{~min}\right)$, we explain the increase of coercivity calling upon firstly apparition of $\mathrm{Cu}$ nucleus, followed by the beginning of the formation of borides [13]. As a result, the relevant region allowing confrontation with the quantitative model of $K_{\text {me }}$ is $f<0.65$.

\section{Modeling of $K_{\text {me }}$}

Magnetoelastic energy due to reversing of $\mathrm{CV}$ is evaluated looking at the costly situation featured by $\mathrm{CV}$ and $\mathrm{AD}$ perpendicularly magnetized $(\theta=\pi / 2$, AD magnetized along $\mathrm{O} z$ ). $\mathrm{CV}$ is supposed to switch when surrounded by the DW, and we will thus pay attention to the case $z_{\mathrm{d}}=L_{0}$. The situation involves three elastically identical media, i.e. AD (superscript d), DW (superscript w) and CV (superscript c) with magnetostriction coefficient $\lambda_{\mathrm{s}}$ measured by SAMR method [14], plotted on Fig. 6 and fitted by Eq. (8),

$$
\lambda_{\mathrm{s}}(f)=24.5 \times 10^{-6}-31.5 \times 10^{-6} f
$$

Young modulus $E$ is calculated in the frame of an effectivemedium theory [8], with nanocrystals $\left(E_{\mathrm{c}}=210 \mathrm{GPa}\right)$ embedded in amorphous residual phase $\left(E_{\mathrm{a}}=160 \mathrm{GPa}\right.$ [12]). One notices that it was not possible to reproduce evolution reported in Ref. [12], even introducing an interfacial medium elastically weak as proposed in Ref. [15] according to Ref. [16]. We finally introduce Poisson's ratio $v \approx 0.33$ according to Ref. [17] and shear modulus $G=0.5 E /(1+v)$.
DW and reversing CV $(\theta=\pi / 2)$ feature the same equilibrium strains $\lambda_{\mathrm{e} x} ;{ }^{\mathrm{c}}=\lambda_{\mathrm{s}}$, and $\lambda_{\mathrm{ey}} ;{ }^{\mathrm{c}}=\lambda_{\mathrm{e} z} ;{ }^{\mathrm{c}}=-\lambda_{\mathrm{s}} / 2$, which differ from those of $\mathrm{AD}$, i.e. $\lambda_{\mathrm{e} y} ;{ }^{\mathrm{d}}=\lambda_{\mathrm{s}}$, $\lambda_{\mathrm{e} x},{ }^{\mathrm{d}}=\lambda_{\mathrm{e} z},{ }^{\mathrm{d}}=-\lambda_{\mathrm{s}} / 2$. As a result, media experiment compressive stresses $\sigma_{j},{ }^{i}$ and shearing stresses $\tau_{j}{ }^{i}$, related to strains $\lambda_{j}{ }^{i}$, and $\gamma_{j}{ }^{i}$, by the way of generalized Hooke's laws are written following [18] in Eq. (9) as:

$\sigma_{x},^{i}-v\left(\sigma_{y},{ }^{i}+\sigma_{z}{ }^{i}\right)=E\left(\lambda_{x},{ }^{i}-\lambda_{\mathrm{e} x}{ }^{i}\right), \quad \tau_{x}{ }^{i}=2 G \gamma_{x}{ }^{i}$

( $i=\mathrm{c}, \mathrm{w}$ or $\mathrm{d}$, cyclic permutations of $x, y, z)$.

This leads to the volumetric magntoelastic density of energy stored in each medium $\mathrm{W}^{i}$ :

$$
W^{\mathrm{i}}=\frac{E}{2(1+v)}\left[\frac{v}{1-2 v}\left(\sum_{j} \Delta \lambda_{j}^{i}\right)^{2}+\sum_{j}\left(\Delta \lambda_{j}^{i}\right)^{2}+2 \sum_{j} \gamma_{j}^{i^{2}}\right]
$$

$\Delta \lambda_{j}^{i}=\lambda_{j}^{i}-\lambda_{\mathrm{e} j}^{i}$

A basic simple hypothesis is that domains impose strains everywhere, i.e. inside DW and CV. Energy variations are localized in $\mathrm{CV}$, allowing to identify $K_{\mathrm{me}}$ as $W^{\mathfrak{C}}$ obtained from Eq (10) and (11) and plotted in Fig. 5,

$$
W^{\mathrm{c}}(\pi / 2)-W^{\mathrm{c}}(0)=K_{\mathrm{me}}=p 9 E \lambda_{\mathrm{s}}^{2} /[4(1+v)],
$$

where $p$ is a numerical prefactor equaling 1 in this first approach. Regarding experimental data, calculated $K_{\text {me }}$ appears strongly overestimated. We thus improve our modelization as follows

In the first step, CV not considered yet, we introduce two identical buffer zones (BZ) (superscript b) at either side of DW, allowing a progressive matching of strain between DW and AD. BZ strains are calculated assuming linear variations from those of DW (now adjustable but assumed still uniform) to those of $\mathrm{AD}$ (equilibrium strains), the thickness $\alpha L_{0}$ of BZ being adjustable too. Magnetization of $\mathrm{BZ}$, controlled by ferromagnetic exchange interactions,

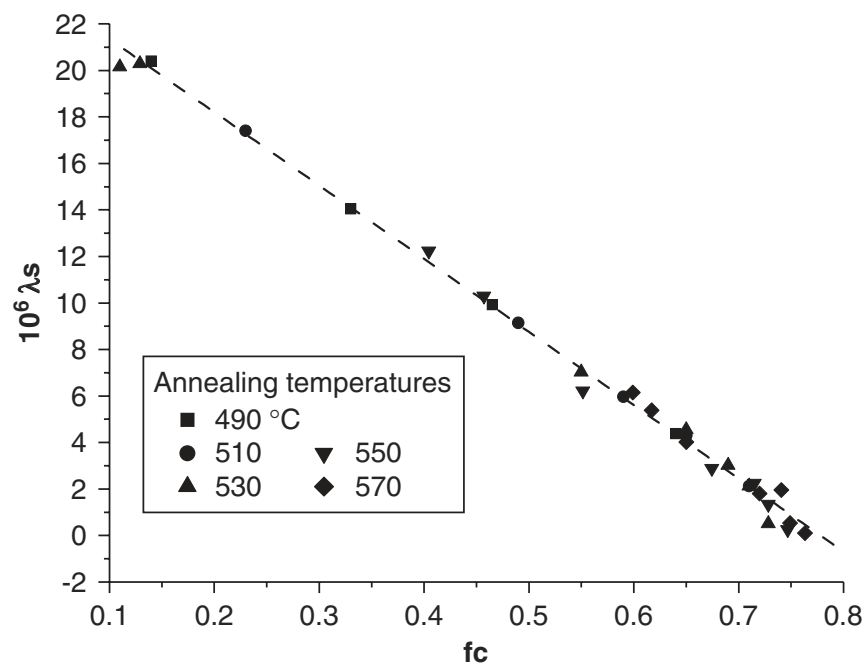

Fig. 5. Experimental values of magnetostriction coefficient $\lambda_{\mathrm{s}}$ corresponding to the database. Superimposed, the linear fit (dotted line) $\lambda_{\mathrm{s}}(f)=\left[\left(24.5 \times 10^{-6}\right)-\left(31.5 \times 10^{-6}\right)\right] f$. 


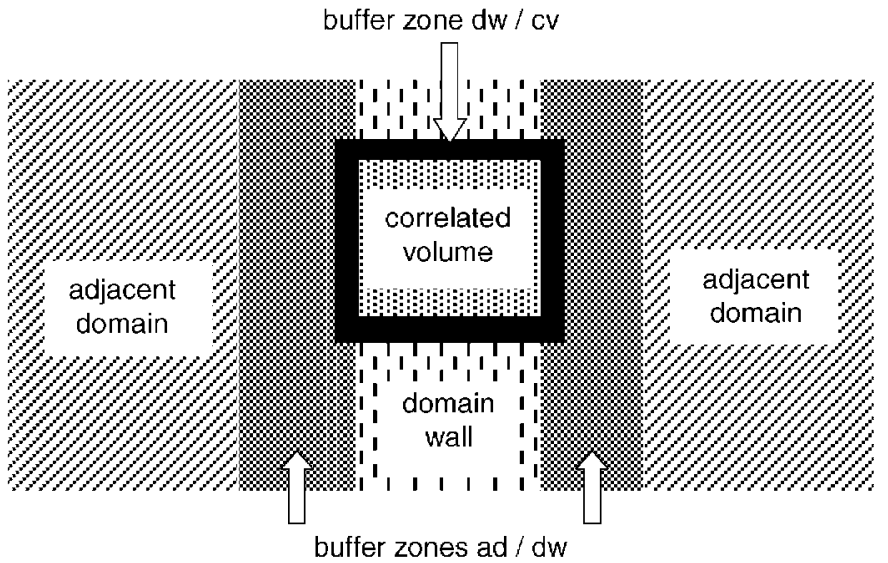

Fig. 6. Context experienced by a reversing $\mathrm{CV}$ with different buffer zones accommodating frustrations. Only the buffer zones $\mathrm{AD} / \mathrm{DW}$ are taken into account in the present work.

follows the bisector of DW and AD magnetizations. Optimal values of adjustable parameters are numerically obtained to minimize the total magnetoelastic energy stored both in DW and BZ. It can be highlighted that calculated strains are still volume conservative and lead to a decrease of energy by $24 \%$ with $\alpha=0.44$.

In the second step, strains featured by DW and BZ being now fixed according to calculated values, we now look at $\mathrm{CV}$ and consider that its strain is imposed by DW. As a result, the initial state $\theta=0$ experiments stress with a resulting energy $\mathrm{W}^{\mathrm{c}}(0)>0$. Moreover, the final state $\theta=$ $\pi / 2$ is more comfortable with BZ than without BZ. All of this leads to a decrease of the prefactor $p$ introduced in Eq. (11), which falls to 0.46 .

In spite of significant reduction, $K_{\mathrm{me}}$ is still overestimated. In fact, shearing strains seem to be idle, leading to an energetically costly concentration of compressive stress. This is due to the high and physically unjustified symmetry featured, i.e. parallepipedic shapes of DW and magnetization direction along edges. Thus, we now consider a DW magnetized along $\mathbf{u}_{x}+\mathbf{u}_{y}$ in the reference frame shown on Fig. 1, with magnetizations of AD along $\mathbf{u}_{x}-\mathbf{u}_{y}$, and $\mathbf{u}_{y}-\mathbf{u}_{x}$ still perpendicular to it. This implies that for each medium, Hooke's laws (Eq. (10)) are now written in local reference frames linked to the direction of magnetization in each medium, equations for continuity of strains involving absolute reference frame and classical transformations to express strains from a medium to another one.

As expected, all adjustable parameters are now used with numerical value of $p$ falling to 0.3 (cf. Fig. 5).

A next step in improvement could consist in adding a second buffer zone (cf Fig. 6) in charge of accommodating mismatch between CV and DW. According to Ref. [8], one can expect a new significant decrease in magnetostatic energy with a much better agreement between experiment and model.

\section{Conclusion}

The present work investigates the way a nanocrystalline soft material develops coherent stress at the scale of, a condition required to obtain a noticeable effect on coercivity from magnetoelastic origin. The reversal mechanism itself is a good candidate, leading us to study the $\mathrm{CV}$ reverse in connection with the macroscopic magnetization process featured by DW displacement. The role of magnetostatics is underlined, with questions still open on the way to include it in a quantitative tractable approach; this problem shared by all the materials exhibiting vanishing anisotropies [19]. As a result, an evolution of the RAM is proposed and used to extract magnetoelastic contribution from $H_{\mathrm{c}}$ measurements provided by a large database offered for investigations. Comparison with model is not perfect; correct evaluation involving numerous degrees of freedom to express the natural behaviour of any physical system is required to take advantage of all possibilities to minimize its energy.

\section{References}

[1] X.Y. Xiong, K.Y. Ho, J. Appl. Phys. 77 (1995) 2094.

[2] K. Suzuki, G. Herzer, J.M. Cadogan, J. Magn. Magn. Mater. 177-181 (1998) 949.

[3] G. Herzer, IEEE Trans. Magn. 25 (1989) 3327.

[4] G. Herzer, IEEE Trans. Magn. 26 (1990) 1397.

[5] G. Herzer, J. Magn. Magn. Mater. 112 (1992) 258.

[6] H. Chazal, O. Geoffroy, J.L. Porteseil, T. Waeckerlé, J. Magn. Magn. Mater. 272-276 (2004) 1436.

[7] H.R. Hilzinger, H. Kronmüller, J. Magn. Magn. Mater. 2 (1976) 11.

[8] H. Chazal, O. Geoffroy, J.L. Porteseil, T. Waeckerlé, Proceedings of SMM 16, Düsseldorf, Vol. 2, p. 555.

[9] P. Rhodes, G. Rowlands, Proceedings of the Leeds Philosophical Literature Society, Vol. 6, 1954, p. 191.

[10] S. Chikazumi, Physics of Magnetism, Wiley, New York, 1978, p. 21 and 217.

[11] G. Herzer, Phys. Scripta T49 (1993) 307.

[12] J. Gutierrez, P. Gorria, J.M. Barandiaran, A. Garcia-Arribas, Nanostructured and Non-Crystalline Materials, in: M. Vazquez, A. Hernando (Eds.), World Science Publications, Singapore 1995, p. 500 .

[13] T. Kulik, A. Hernando, M. Vazquez, J. Magn. Magn. Mater. 133 (1994) 310.

[14] F. Alves, P. Houée, M. Lécrivain, F. Mazaleyrat, J. Appl. Phys. 81 (8) (1997) 4322.

[15] C.W. Nan, J.H. Huang, G.J. Weng, J. Magn. Magn. Mater. 233 (2004) 219.

[16] M.D. Kluge, D. Wolf, J.F. Lutsko, S.R. Philipot, J. Appl. Phys. 67 (1990) 2370.

[17] D.-X. Chen, L. Pascual, F.J. Castanio, M. Vasquez, A. Hernando, IEEE Trans. Magn. 37 (2001) 994.

[18] K. Delaere, W. Heylen, R. Belmans, K. Hameyer, European Phys. J. Appl. Phys. 13 (2001) 115.

[19] T. Waeckerlé, H. Fraisse, J. Magn. Magn. Mater. 215-216 (2000) 184. 